\title{
Fuelling (and quenching) star formation in Brightest Cluster Galaxies
}

\section{S. I. Loubser*}

Centre for Space Research, North-West University, Potchefstroom 2520, South Africa

E-mail: ilani.loubserenwu.ac.za

\section{A. Ratsimbazafy}

Centre for Space Research, North-West University, Potchefstroom 2520, South Africa

E-mail: ando.ratsimbazafy@nwu.ac.za

\begin{abstract}
We have obtained detailed, spatially-resolved SALT spectroscopy over the entire optical wavelength range for eight nearby, active brightest cluster galaxies (BCGs) in X-ray luminous groups and clusters. This enables us to derive fundamental gas properties such as electron density and gas temperature, which in turn, enables us to derive accurate abundances. Our small, carefully selected, sample consists of BCGs with detected $\mathrm{H} \alpha$ filaments, and have existing data from the $\mathrm{X}$-ray regime available, but lacks the detailed optical emission-line data that will probe the dominant ionisation processes, excitation sources, morphology and kinematics of the hot gas. This, combined with the other multi-wavelength data, will form a complete view of the different phases (hot and cold gas and young stars) and how they interact in the processes of star formation and feedback detected in central galaxies in cooling flow systems, as well as the influence of the surrounding intracluster medium (ICM). Here, we present our first results, illustrating the complexity and spatial variation of the ionisation mechanisms in the nuclei, as well as the large variety of hot gas properties in BCGs.
\end{abstract}

SALT Science Conference 2015

1-5 June 2015

Stellenbosch Institute of Advanced Study, South Africa

* Speaker. 


\section{Introduction}

The most massive of early-type galaxies - brightest cluster galaxies (BCGs) - are unique with their extremely high luminosities, diffuse and extended structures, and dominant central locations in clusters. They are believed to be sites of very interesting evolutionary phenomena (e.g. dynamical friction, galactic cannibalism, cooling flows). These processes leave different imprints in the dynamical properties, the detailed chemical abundances, and the star formation histories of these galaxies, which can be studied using high-quality spectroscopy [16].

A fraction of the BCGs exhibit evolutionary histories that are in stark contrast with the conventional expectation that giant elliptical galaxies in clusters are all quiescent, "red and dead" systems. BCGs, particularly those residing at the centres of cool-core clusters exhibit "blue cores" corresponding to the presence of a small fraction of young stars [2,21], and reveal activity in their radio emission, have optical emission-line nebulae, excess UV light, far-infrared emission from warm dust, and molecular hydrogen [10]. BCGs in the nearby Universe exhibit diverse star formation histories [18], despite the fact that most are located in a similar, privileged environment in the centres of X-ray luminous clusters.

Cooling-flow clusters are common in the local Universe and BCGs are often found at the centres of these systems. If the central cluster density is high enough, intracluster gas can condense and form stars at the bottom of the potential well. The lack of widespread detection of iron lines expected from cluster gas cooling in X-ray observations of cool-core clusters contradicted the model that many young stars are formed in cooling flows (this is sometimes referred to as the "cooling flow problem"). The central cluster galaxies often host radio-loud AGN which may account for the necessary heating to counteract radiative cooling [27]. Other mechanisms have also been proposed to explain where cooling flow nebulae obtain their energy: fast shocks [3], self-irradiation by the cooling hot gas [8], turbulent mixing layers etc. The dense core of a galaxy cluster represents a unique environment, where the hot ICM is cooling most rapidly, feedback from the AGN is most effective, and the BCG dominates the mass. This represents one of the few places in the Universe where large-scale cooling and feedback processes can be readily observed.

Gas accretion models in the centres of clusters have received a lot of attention lately, e.g. the "cold-gas accretion model" which has emerged as a useful framework for interpreting various observed properties of elliptical galaxies as well as BCGs in the centres of clusters [26, 22, 18]. This SALT project is concerned with the optical emission line properties of the reheated gas that ultimately causes the cooling-feedback cycle to repeat. The heating source(s) in BCGs currently remains uncertain as no proposed heating mechanism reproduces all the emission-line properties within the optical wavelength ranges previously observed. A single dominant mechanism may not apply to all BCG nebulae, and there may be a mixture of heating mechanisms acting within a single nebula $[13,11]$. Thus to get more information, which will enable the dominant mechanism(s) to be identified, we measured more line ratios over the entire optical wavelength range, using SALT.

Our targets have been selected from the literature to have $\mathrm{H} \alpha$ detections and strong indications of star formation activity [9]. A complete view of the star formation and feedback processes necessarily incorporates the stars with the gas and an understanding of the processes by which these phases interact, and therefore, requires information from several wavelength regimes. This new optical spectroscopic data, combined with the other existing multiwavelength data (Represen- 


\begin{tabular}{|c|c|c|c|c|c|}
\hline BCG Name & $z$ & $\alpha_{J 2000}$ & $\delta_{J 2000}$ & $\begin{array}{c}\mathrm{E}(\mathrm{B}-\mathrm{V})_{g a l} \\
(m a g)\end{array}$ & $\begin{array}{l}\text { Exp. time } \\
(s)\end{array}$ \\
\hline 2MASXJ23194046-7313366 & 0.098 & $23 \mathrm{~h} 19 \mathrm{~m} 40.9 \mathrm{~s}$ & $-73 \mathrm{~d} 13 \mathrm{~m} 38 \mathrm{~s}$ & 0.031 & $\begin{array}{l}16600 \mathrm{~B} \\
12760 \mathrm{R}\end{array}$ \\
\hline 2MASXJ13025254-0230590 & 0.085 & $13 \mathrm{~h} 02 \mathrm{~m} 52.6 \mathrm{~s}$ & $-02 \mathrm{~d} 30 \mathrm{~m} 59 \mathrm{~s}$ & 0.023 & $\begin{array}{l}10640 \mathrm{~B} \\
5480 \mathrm{R}\end{array}$ \\
\hline ESO248-006 & 0.076 & $03 \mathrm{~h} 17 \mathrm{~m} 57.7 \mathrm{~s}$ & $-44 \mathrm{~d} 14 \mathrm{~m} 17 \mathrm{~s}$ & 0.012 & $\begin{array}{l}8295 \mathrm{~B} \\
6400 \mathrm{R}\end{array}$ \\
\hline ESO541-013 & 0.057 & $01 \mathrm{~h} 02 \mathrm{~m} 41.7 \mathrm{~s}$ & $-21 \mathrm{~d} 52 \mathrm{~m} 55 \mathrm{~s}$ & 0.018 & $\begin{array}{l}1850 \mathrm{~B} \\
1020 \mathrm{R} \\
2000 \mathrm{ER}\end{array}$ \\
\hline Hydra A & 0.055 & $09 \mathrm{~h} 18 \mathrm{~m} 05.7 \mathrm{~s}$ & $-12 \mathrm{~d} 05 \mathrm{~m} 44 \mathrm{~s}$ & 0.042 & $7317 \mathrm{R}$ \\
\hline ESO349-010 & 0.049 & $23 \mathrm{~h} 57 \mathrm{~m} 00.7 \mathrm{~s}$ & $-34 d 45 \mathrm{~m} 33 \mathrm{~s}$ & 0.014 & $\begin{array}{l}7800 \mathrm{~B} \\
2700 \mathrm{R}\end{array}$ \\
\hline 2MASXJ12571157-1724344 & 0.047 & $12 \mathrm{~h} 57 \mathrm{~m} 11.6 \mathrm{~s}$ & $-17 \mathrm{~d} 24 \mathrm{~m} 34 \mathrm{~s}$ & 0.069 & $\begin{array}{l}8250 \mathrm{~B} \\
2200 \mathrm{R}\end{array}$ \\
\hline MCG -02-12-039 & 0.033 & $04 \mathrm{~h} 33 \mathrm{~m} 37.8 \mathrm{~s}$ & $-13 \mathrm{~d} 15 \mathrm{~m} 43 \mathrm{~s}$ & 0.136 & $2600 \mathrm{R}$ \\
\hline
\end{tabular}

Table 1: Objects observed with optical spectroscopy. The galactic extinction, $\mathrm{E}(\mathrm{B}-\mathrm{V})_{\text {gal }}$, is described in the text. The rest-frame wavelength coverage is $\sim 3500-6000 \AA$ for the blue (B), $\sim 5000-7500 \AA$ for the red (R), and $\sim 6100-8900 \AA$ for the extreme red (ER).

tative XMM Cluster Structure Survey REXCESS, GALEX etc.), will form a complete view of the different phases (hot and cold gas and young stars) and how they interact in the processes of star formation and feedback detected in central galaxies in cooling flow clusters, as well as the influence of the host cluster environment. Throughout this paper we adopt a $\Lambda$ CDM cosmology with $\Omega_{m}=0.3, \Omega_{\Lambda}=0.7$, and $H_{0}=70 \mathrm{~km} \mathrm{~s}^{-1} \mathrm{Mpc}^{-1}$.

\section{SALT data}

The data was obtained with the Robert Stobie Spectrograph (RSS) on the SALT telescope between the 2013-1 and 2014-2 observing semesters. The PG0900 grating, with a 1.5" slit placed on the galaxy's major axis, was used at various grating angles to cover the entire optical wavelength range. In addition, the necessary flat-field and arc frames were also observed at regular intervals, as well as spectrophotometric standard stars for flux calibration.

The basic reductions were done using the PySALT: SALT science pipeline ${ }^{1}$ [6], whilst further reductions were done in IRAF $^{2}$. The data reduction methodology is described in detail in [19]. The individual exposures were carefully combined to make a blue and red 2-D frame for each galaxy. The galaxy and propagated error spectra were binned in the spatial direction to ensure a minimum signal-to-noise ratio $(\mathrm{S} / \mathrm{N})$ of 10 per bin in the red frame. The same bins were then extracted from

\footnotetext{
${ }^{1}$ http://pysalt.salt.ac.za/

${ }^{2}$ IRAF is distributed by the National Optical Astronomy Observatories, which are operated by the Association of Universities for Research in Astronomy Inc., under cooperative agreement with the National Science Foundation.
} 
the blue frames, if applicable, to ensure identical spatial locations of the bins in the blue and red frame. The $\mathrm{S} / \mathrm{N}$ ratios of the bins in the blue frame are typically $>10$. The $\mathrm{S} / \mathrm{N}$ ratios were chosen to resolve the optimal number of possible measurement bins whilst maintaining acceptable errors on the measurements. As a result, the spatial bins become wider with increasing radius from the centre of the galaxy, typically reaching up to $20 \mathrm{kpc}$ on both sides from the centre of the galaxy.

To measure the emission lines, we use a combination of the PPXF [5] and GANDALF [24] routines $^{3}$. GANDALF enables a (total) reddening correction to be included, and propagates errors in the measurements. All the stars of the MILES stellar library [23] were used to construct optimal stellar absorption templates. After the kinematics are fixed, following the procedure in [24] and [17], a Gaussian template is constructed for each emission line at each iteration, and the best linear combination of both stellar and emission-line templates (with positive weights) is determined.

The $\mathrm{H} \alpha$ and [NII] lines did not require deconvolution due to the relatively high spectral resolution, and were simultaneously fit using a combination of three Gaussians. There are cases where the [SII] lines are significantly broadened due to gas kinematics or other mechanisms and not well approximated by a single Gaussian for each line. Additional broad Gaussian components were then added for a better fit to the observed lines. See Figure 1b (2MASXJ23194046-7313366), for an example of broad [SII] lines.

We compare our emission line measurements for 2MASXJ23194046-7313366 with those of [9]. From the single central spectrum, they measured an $\mathrm{H} \alpha$ equivalent width of $10.8 \pm 1.4 \AA$, a [NII] $\lambda 6584 / \mathrm{H} \alpha$ ratio of $1.2 \pm 0.1$ and an $\mathrm{H} \alpha \mathrm{FWHM}$ of $12.3 \pm 0.5 \mathrm{~km} \mathrm{~s}^{-1}$. Our measurements are $9.79 \pm 0.03 \AA, 1.03 \pm 0.01$ and $13.5 \pm 0.5 \mathrm{~km} \mathrm{~s}^{-1}$, respectively, and comparable to the earlier measurements.

\section{Gas properties}

\section{Extinction}

The Galactic contribution to the total extinction was derived for each galaxy using the maps of [25]. The total extinctions were derived using the $\mathrm{H} \beta$ and $\mathrm{H} \gamma$ (in the blue frames), as well as with $\mathrm{H} \beta$ and $\mathrm{H} \alpha$ (in the red frames) relative strength ratios. The difference between the total extinction and Galactic extinction is attributed to the internal extinction by the BCG. For case $\mathrm{B}$ recombination $\left(\mathrm{n}_{e}\right.$ of $100 \mathrm{~cm}^{-3}$, and $\mathrm{T}_{e}$ of $10000 \mathrm{~K}$ ), the unattenuated value of the Balmer decrement is $\mathrm{I}(\mathrm{H} \alpha) / \mathrm{I}(\mathrm{H} \beta)=2.86$, and $\mathrm{I}(\mathrm{H} \gamma) / \mathrm{I}(\mathrm{H} \beta)=0.47$. We adopt the dust model of [4] to measure the flux attenuation values at the desired wavelength for any given $\mathrm{E}(\mathrm{B}-\mathrm{V})$ value. Our data provides new estimates on the amount of intrinsic reddening in the optical nuclei of the BCGs of cool core clusters.

\section{BPT-diagram}

BPT diagrams [1] such as $\mathrm{I}([\mathrm{OIII}] \lambda 5007) / \mathrm{I}(\mathrm{H} \beta)$ vs $\mathrm{I}([\mathrm{NII}] \lambda 6584) / \mathrm{I}(\mathrm{H} \alpha)$, $\mathrm{I}([\mathrm{OIII}] \lambda 5007) / \mathrm{I}(\mathrm{H} \beta)$ vs I([SII] $\lambda \lambda 6717,6731) / \mathrm{I}(\mathrm{H} \alpha), \mathrm{I}([\mathrm{OIII}] \lambda 5007) / \mathrm{I}(\mathrm{H} \beta)$ vs $\mathrm{I}([\mathrm{OI}] \lambda 6300) / \mathrm{I}(\mathrm{H} \alpha)$ can be constructed for emission line galaxies to separate ionisation from AGN and star formation.

\footnotetext{
${ }^{3}$ We make use of the corresponding PPXF and GANDALF IDL (Interactive Data Language) codes which can be retrieved at http:/www.leidenuniv.nl/sauron/.
} 


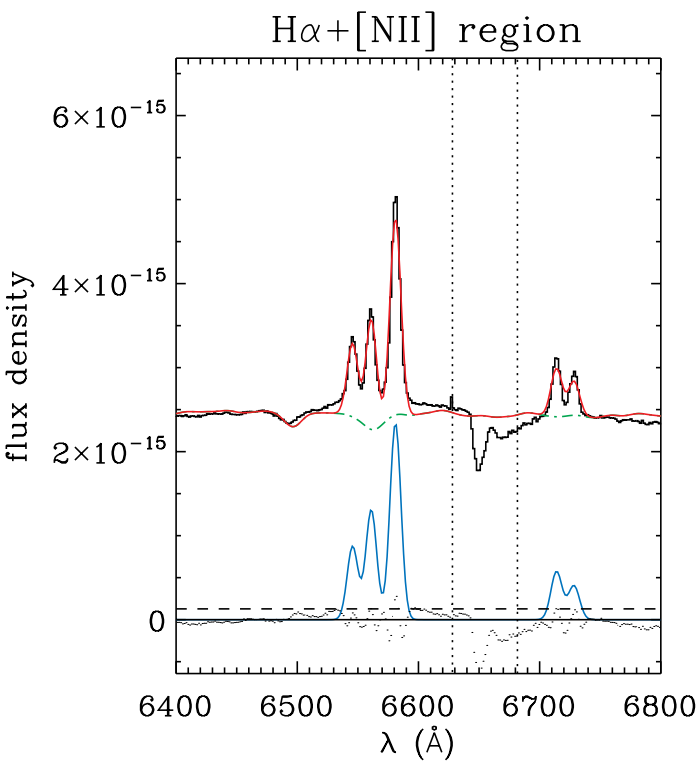

(a) MCG -02-12-039

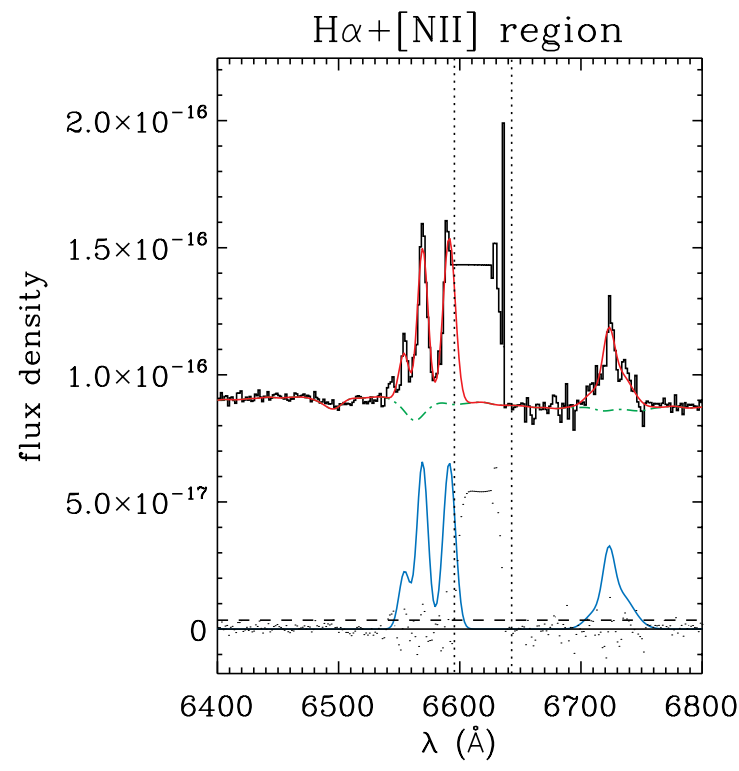

(b) 2MASXJ23194046-7313366

Figure 1: Examples of Gaussian fitting to MCG -02-12-039 and 2MASXJ23194046-7313366 in the region of $\mathrm{H} \alpha$ and [NII]. The red line indicates the best-fitting stellar template and Gaussians at the emission lines. The green line indicates the best-fitting stellar templates with the emission lines subtracted. The blue line indicates the flux density for the measured emission lines. Residuals from the fit are also shown in the bottom panel. Doted vertical lines are masked from the fit (for example saturated sky absorption lines, CCD gaps, etc). Lines from left to right are: [NII] $\lambda 6548$, $\mathrm{H} \alpha,[\mathrm{NII}] \lambda 6584,[\mathrm{SII}]$ doublet. These figures also illustrate that single, narrow Gaussians are not always sufficient to approximate the emission lines, and that additional broad Gaussian components or added "wings" are sometimes needed.

[15] used a combination of photo-ionisation and stellar population synthesis models to place a theoretical upper limit on the location of star forming galaxies on the BPT diagram. Thus, the galaxies above the [15] criterium are defined as purely AGN. [14] revised the criterium to a curve below which purely star forming galaxies are found. Thus, the area between the curves contain the composite objects. We show two BPT diagrams for 2MASXJ12571157-1724344, as an example, in Figure 2. Seeing can account for $\sim 1$ arcsec spread in the data points, nevertheless, a clear trend can be seen as the bins are located further from the nucleus of the galaxy. AGN-like line ratios can also be caused by other heating mechanisms (see Figure 14 in [20]), and the ionisation regions require more detailed modelling to disentangle the complex combination of AGN feedback, photoionisation from hot stars, and other mechanisms.

\section{Electron gas density and temperature}

Doublet line ratios such as I([OII] $\lambda 3726) / \mathrm{I}([\mathrm{OII}] \lambda 3729)$, or I([SII] $\lambda 6716) / \mathrm{I}([\mathrm{SII}] \lambda 6731)$ are very useful measures of electron densities (in a low-ionisation scenario). These densities, $\mathrm{n}_{e}$, are typically observed to be 100 to $300 \mathrm{~cm}^{-3}$, and are very weakly dependent on electron temperature. 

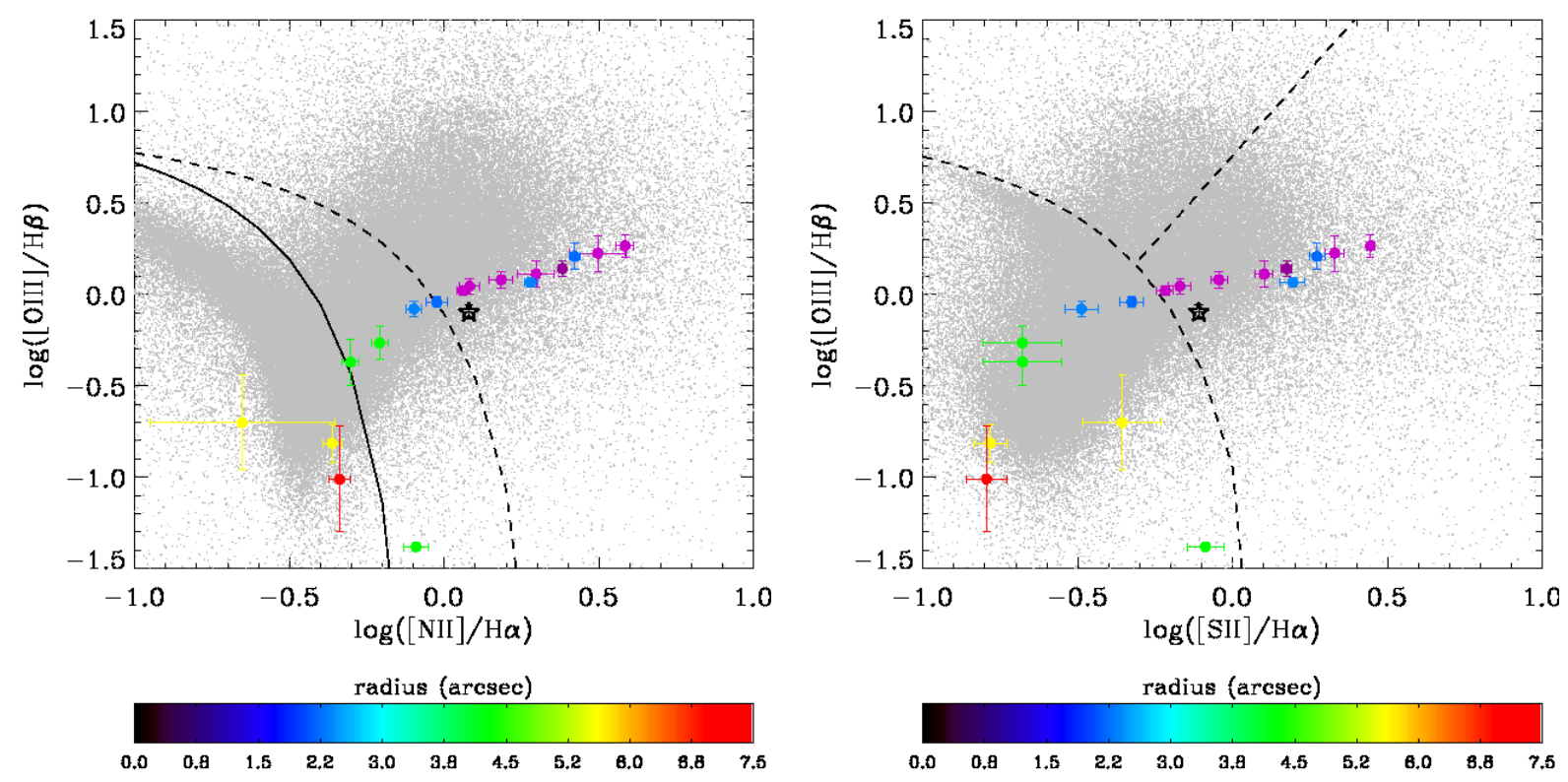

Figure 2: BPT diagrams for 2MASXJ12571157-1724344. Left: $\log ([\mathrm{OIII}] \lambda 5007 / \mathrm{H} \beta)$ vs $\log ([\mathrm{NII}] \lambda 6584 / \mathrm{H} \alpha)$. Right: $\log ([\mathrm{OIII}] \lambda 5007 / \mathrm{H} \beta)$ vs $\log ([\mathrm{SII}] \lambda \lambda 6717,6731 / \mathrm{H} \alpha)$. The black star indicates the measurement across the whole galaxy (one single extracted spectrum). The grey points are galaxies from the Sloan Digital Sky Survey [14]. The solid line represents the separation between HII regions and AGN as defined by [14], while the dashed line separates HII regions and AGN as defined by [15] (see text).

The density equation is iteratively solved using a technique originally developed by [7], applied with the NEBULAR package in STSDAS, layered on the IRAF environment.

Another fundamental parameter for the derivation of all other physical and chemical quantities in a photoionised diffuse plasma is the electron temperature, since virtually all the observables are strong functions of temperature. The most common means of estimating the electron temperature uses two emission lines of the same element which have different thresholds for collisional excitation.

Electron temperature can be measured from the $\mathrm{I}([\mathrm{NII}] \lambda 6548+[\mathrm{NII}] \lambda 6583) / \mathrm{I}([\mathrm{NII}] \lambda 5755)$ and $\mathrm{I}([\mathrm{SII}] \lambda 6716+[\mathrm{SII}] \lambda 6730) / \mathrm{I}([\mathrm{SII}] \lambda 4068+[\mathrm{SII}] \lambda 4076)$ ratios (depending on the ionisation scenario), and is also very weakly dependent on electron density. We show the [OII] and [NII] line ratios and their associated electron densities and temperatures as a function of radius for 2MASXJ12571157-1724344, as an example, in Figure 3.

\section{Other diagnostic line measurements}

Many more model independent properties can be derived, e.g. the helium recombination lines provide a model-independent way to check the hardness of the incident continuum. Metallicities can be estimated using $\mathrm{R}_{23}=(\mathrm{I}([\mathrm{OII}] \lambda 3727)+\mathrm{I}([\mathrm{OIII}] \lambda 4959+[\mathrm{OIII}] \lambda 5007)) / \mathrm{I}(\mathrm{H} \beta)$, where additional line ratios, e.g. I([NII] $\lambda 6583) / \mathrm{I}([\mathrm{OII}] \lambda 3727)$ are required to break the degeneracy. An instantaneous measure of star formation, the $\mathrm{H} \alpha$ star formation rate can be measured, and the 


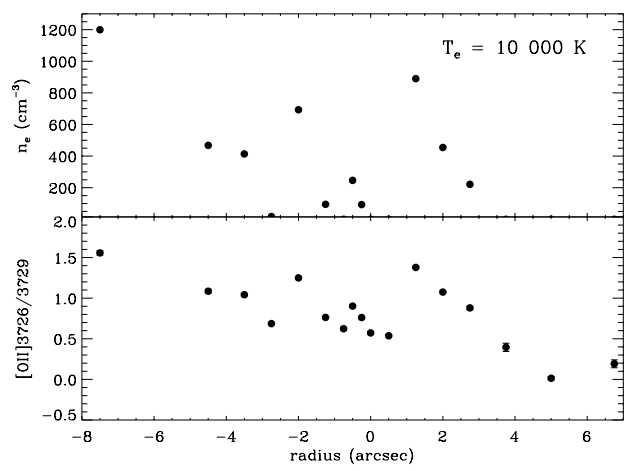

(a) Line ratios and electron gas density

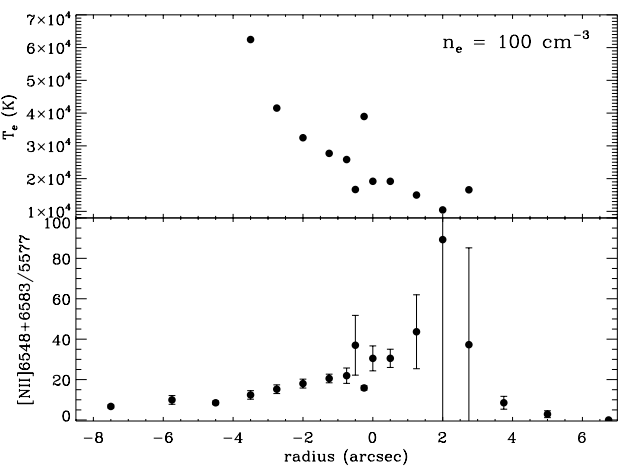

(b) Line ratios and electron gas temperature

Figure 3: Radial variation in [OII] and [NII] ratio measurements, electron density and temperature of the gas in 2MASXJ12571157-1724344.

[OIII] $] 4363 \AA$ Aine can be used as an indicator for shocks.

\section{Results summary and conclusions}

There are several possible sources of ionisation in the cool cores of galaxy clusters. The most popular ideas have been: (a) the central AGN, (b) young stellar populations, (c) X-rays from the ICM, (d) heat conduction from the ICM to the cold filament, (e) shocks and turbulent mixing layers, and (f) collisional heating by cosmic rays.

Our SALT data, collected over two years, enables us to measure all the emission lines (with amplitude-to-noise ratio $>2$ ) in the optical wavelength range. Our initial calculations of the hot gas properties illustrate a variety of gas kinematics and ionisation mechanisms as well as a change in hot gas properties further from the galaxy nucleus. We interpret this variety in hot gas properties as different snapshots of BCGs at different stages of the cooling-feedback cycle.

Up to this point, the measurements and the resulting calculations are model independent, however photoionization models such as CLOUDY can now be used to model the data [12]. If we use the BPT diagrams, but now include model expectations for star formation, cooling plasma, shocks, conduction, and collisional ionisation by cosmic rays, then these complex systems can be better understood.

Placing the hot gas ionisation mechanisms into context with all available data from X-ray (ICM) through radio, should allow for a more robust solution to this long-standing problem, while combining the hot gas kinematics with deep X-ray and radio studies may shed new light on the motions of this gas.

\section{Acknowledgments}

SIL and AR are financially supported by the South African National Research Foundation. All of the observations reported in this paper were obtained with the Southern African Large Telescope 
(SALT) under program 2013-1-RSA_OTH-011, 2013-2-RSA_OTH-002, 2014-1-RSA_OTH-003, 2014-2-SCI-014 (PI: Ilani Loubser).

\section{References}

[1] Baldwin J. A., Phillips M. M., Terlevich R., 1981, PASP, 93, 5

[2] Bildfell C., Hoekstra H., Babul A., Mahdavi A., 2008, MNRAS, 389, 1637

[3] Binette L., Dopita M. A., Tuohy I. R., 1985, ApJ, 297, 476

[4] Calzetti D., Armus L., Bohlin R. C., Kinney A. L., Koornneef J., Storchi-Bergmann T., 2000, ApJ, 533,682

[5] Cappellari M., Emsellem E., 2004, PASP, 116, 138

[6] Crawford, S. M., Still, M., Schellart, P., et al., 2010, in SPIE Conference Series, Vol. 7737, 25

[7] De Robertis M. M., Dufour R. J., Hunt R. W., 1987, JRASC, 81, 195

[8] Donahue M., Voit G. M., 1991, ApJ, 381, 361

[9] Donahue M. et al., 2010, ApJ, 715, 881

[10] Donahue M. et al., 2015, ApJ, 805, 177

[11] Edwards L. O. V., Robert C., Molla M., McGee S. L., 2009, MNRAS, 396, 1953

[12] Ferland G. J., Korista K. T., Verner D. A., Ferguson J. W., Kingdon J. B., Verner E. M., 1998, PASP, 110,761

[13] Hatch N. A., Crawford C. S., Fabian A. C., 2007, MNRAS, 380, 33

[14] Kauffmann G. et al., 2003, MNRAS, 346, 1055

[15] Kewley L. J., Dopita M. A., Sutherland R. S., Heisler C. A., Trevena J., 2001, ApJ, 556, 121

[16] Loubser, S. I. \& Soechting, I. K., 2013, MNRAS, 431, 2933

[17] Loubser, S. I., 2014, MNRAS, 439, 416

[18] Loubser, S. I., Babul, A., Hoekstra, H., Mahdavi, A., Donahue, M., Bildfell, C., and Voit, G. M., 2016, MNRAS, 456, 1565

[19] Loubser, S. I., Donahue, M., Voit, G.M., 2015, in proceedings of SALT Science conference, POS (SSC2015) 068

[20] McDonald, M., Veilleux, S., Rupke, D. S. N., 2012, ApJ, 746, 153

[21] Pipino A., Kaviraj S., Bildfell C., Babul A., Hoekstra H., Silk J., 2009, MNRAS, 395, 462

[22] Prasad D., Sharma P., Babul A., 2015, ApJ, 811, 108

[23] Sánchez-Blázquez P. et al., 2006, MNRAS, 371, 703

[24] Sarzi M. et al., 2006, MNRAS, 366, 1151

[25] Schlegel D. J., Finkbeiner D. P., Davis M., 1998, ApJ, 500, 525

[26] Voit G. M., Donahue M., 2015, ApJL, 799, L1

[27] Von der Linden A., Best P.N., Kauffmann G., White S.D.M., 2007, MNRAS, 379, 867 